\title{
Effect of eight weeks of cervical stability exercise on flexion relaxation phenomenon, pain, and disability in patients with non-specific chronic neck pain
}

\section{Hasan Shamsi}

Shahid Beheshti University of Medical Sciences School of Rehabilitation

Khosro Khademi-Kalantari

Shahid Beheshti University of Medical Sciences School of Rehabilitation

Alireza Akbarzade-Baghban

Shahid Beheshti University of Medical Sciences School of Rehabilitation

Neda Izadi

Shahid Beheshti University of Medical Sciences School of Rehabilitation

Farshad Okhovatian ( $\sim$ farshadokhovatian1965@gmail.com )

Shahid Beheshti University of Medical Sciences School of Rehabilitation

\section{Research article}

Keywords: Flexion relaxation phenomenon, Chronic neck pain, Cervical erector spinae, Stability exercise

Posted Date: June 8th, 2020

DOI: https://doi.org/10.21203/rs.3.rs-32737/v1

License: (c) (1) This work is licensed under a Creative Commons Attribution 4.0 International License.

Read Full License 


\section{Abstract}

Background: The effect of therapeutic exercise on cervical flexion relaxation phenomenon (FRP) is questionable. This study aimed at investigating the effect of eight weeks of cervical stability exercise on FRP, pain, and disability in NSCNP patients.

Methods: This quasi-experimental study was conducted on 25 subjects with NSCNP. Surface electromyographic signals of the cervical erector spinae (CES) muscles in a sitting position were recorded before and after the intervention. The occurrence of FRP and flexion relaxation ratio (FRR) were analyzed before and after the eight weeks of cervical stability exercise. The low-load craniocervical flexion exercise was performed by the subjects in crook lying position. In addition, the intensity of pain and disability were assessed via visual analogue index (VAS) and neck disability index (NDI).

Results: The FRR of CES muscles increased after the intervention. Similarly, the occurrence of FRP increased among NSCNP patients after the intervention. The average changes of VAS, NDI, and FRR were not associated with gender and duration of pain. Moreover, VAS and NDI scores significantly decreased after the intervention.

Conclusions: Flexion relaxation phenomenon can be improved via specific stability exercises. The improvement of flexion relaxation phenomenon apparently can pain and disability.

\section{Background}

Chronic neck pain (CNP) is a common musculoskeletal disorder, affecting up to $35-65 \%$ of adult population at some point in their lifetime [1]. CNP can lead to disability, decrease quality of life, impose economic costs, and have a negative impact on patients, families, and health care system [2]. Although, medical history and clinical evaluation can suggest the cause of pain, in most cases underlying pathological cause of the complaint is unclear, so it is labeled as non-specific chronic neck pain (NSCNP) [3]. According to an emerging school of thought, impaired neuromuscular function is the pathologic basis of NSCNP. Previous studies have reported altered muscle activation patterns and impaired neuromuscular function in patients with neck pain; for instance, they reported a decrease in deep and an increase in superficial muscle activation and a disability in activating deep flexor muscles during rapid limb movement [4-6].

Based on the results of previous studies, myoelectric activity of cervical and lumbar erector spinae muscles decreases after $75-80 \%$ of full flexion in healthy subjects, which is known as flexion relaxation phenomenon (FRP) [6, 7]. Transferring the extension moment force from superficial mover muscles to deeper stabilizer muscles and passive structures is the main mechanism that has previously been attributed to FRP [6-8]. Previous studies have shown that cervical FRP in CNP patients starts soon and ends late, as compared with healthy groups. In addition, flexion relaxation ratio (FRR) reduces in patients, as compared with healthy subjects $[6,9,10]$. According to Murphy et al. (2010), FRR is an objective 
assessment method and a reliable marker of neuromuscular impairment that can discriminate CNP patients from healthy subjects [9].

In addition, FRR is a useful clinical tool that not only helps to diagnose impaired neuromuscular function in CNP patients but also can evaluate the effects of therapeutic interventions [11, 12].

In upright postures, the role of the osteoligamentous passive system in stabilizing the cervical spine is minimal (20\%) and the deep segmental muscles play an important role in supporting the cervical lordosis and maintaining the spine postural form (80\%) [13]. Moreover, previous studies have shown that deep cervical flexors (DCF), such as longus colli and longus capitis that resist against destabilizing gravitational forces, are more likely to become dysfunctional in CNP patients $[13,14]$. Furthermore, based on the results of previous studies, not only a reduction in strength and endurance capacity of DCF can lead to CNP, but also a deficient neuromuscular coordination between deep segmental stabilizer muscles and superficial muscles can result in pain $[15,16]$. Therefore, persisted activation of CES in CNP patients is a protective mechanism to increase cervical stabilization, altered motor control strategies and absence of FRP $[6,17]$. Hence, the stabilization exercise program may improve FRR through activating deep segmental stabilizer muscles and reducing the activity of superficial CES.

Neblett et al. (2010) reported that FRP could be improved via treatment. They examined the effect of a rehabilitation program on FRR in patients with chronic low back (CLB) pain. Their program included a progressive functional exercise and counseling sessions to manage pain and stress. Moreover, they trained the patients to relax their back muscles at the end of trunk flexion through biofeedback. After seven weeks of treatment, $60 \%$ of the patients reached normal FRR [18]. In addition, the effect of neurac sling exercise, exercise program using a Swiss ball, and stabilization exercise on FRR in CLB patients have previously been investigated [19-21]. Counter to low back pain (LBP), there are few pieces of evidence about the effect of therapeutic interventions on flexion relaxation parameters in CNP patients. Murphy et al. (2010) assessed the influence of a 4-weeks chiropractic care on FRP in CNP patients and reported a minimal increase in FRR after the treatment [22]. Moreover, another recent study assessed the effect of an 8- week CES muscle stretching technique on FRP in CNP patients and showed a significant improvement in FRR only in the diagonal stretching group [23].

To our knowledge, there is no study assessing the effect of cervical stabilization exercise on FRP parameters. The aim of this study was to evaluate the effect of cervical stability exercise on FRP, VAS, and NDI parameters. As the main hypothesis of our study, it was assumed that the stability exercise can improve FRR, VAS, and NDI.

\section{Methods}

\section{Participants}

In this study, taking into account $25 \%$ mean difference in FRR for bilateral erector spinae between the two groups as well as a-level of 0.05 (type I error) and $\beta$-value of 0.10 (type II error), 25 patients with NSCNP 
and 25 healthy volunteers were recruited. Taking into consideration a type I error of 0.05 , type II error of 0.1 (power $=90 \%$ ), and $\sigma$ of 11,25 adults with non-specific CNP participated in this quasi-experimental study. They were recruited from among those admitted to physical therapy and orthopedic clinics in Tehran province. The patients were examined by an experienced physiotherapist and orthopedic surgeon and diagnosed with NSCNP. Based on the inclusion criteria, the study was conducted on:

1) Patients aged 20 to 45 years.

2) Cases who had experienced persistent pain for at least three months without a specific pathology (e.g. no history of degenerative disease, discopathy, radiculopathy).

3) Cases who without a history of any type of head, shoulder, and lumbar pain during the past two years.

Based on the exclusion criteria, the following cases were excluded from the study:

1) Patients with a pain score more than $5 \mathrm{~cm}$ based on a visual analog scale (VAS).

2) Cases who had participated in a neck rehabilitation program during the past three months.

3) Patients with a history of systemic disease and any type of cervical or shoulder trauma.

The method was approved by the Research Ethics Committee of School of rehabilitation- Shahid Beheshti University of Medical Sciences research committee (approval ID = IR.SBMU.RETECH. REC.1398.405). We explained clearly all steps of study to subjects and wrote informed consent before beginning the study.

\section{Experimental Protocol}

All cases were tested in a 45- min session in a laboratory. Before the test, pain severity was determined using VAS. In addition, the subjects completed NDI questionnaire. Then, each subject was asked to sit on an adjustable stool with hips and knees at an angle of $90^{\circ}$, with feet on the floor positioned shoulder width-apart, with arms relaxed by their side, looking at an eye-level point. Because of the effect of different seated posture on cervical spine alignment, during the test each subject was asked to maintain a neutral lumbar lordosis i.e. the midpoint between full flexion and extension that was determined by a physiotherapist $[24,25]$. Therefore, seated posture was standardized in a neutral lumbar lordosis.

Furthermore, each subject was asked to perform neck full flexion and extension and the mid-position was set as the neutral head position $[25,26]$. The upper thorax was stabilized tightly by a belt at the level of T1-T7 spine to immobilize this region. Before the test, all the cases had become informed about the protocol of the test and the starting neutral position was determined. Then, each subject was asked to maintain a neutral starting position for $4 \mathrm{~s}$ (phase 1), complete cervical full forward flexion for $4 \mathrm{~s}$ (phase 2), sustain full flexion for 4 s (phase 3), and perform re-extension to starting position for 4s (phase 4) [26]. The protocol of the test was performed in three trials. A digital metronome was used to control the effects of speed and cumulative daily loading on FRR, and all the steps of the test were carried out in the 
morning. All parts of the protocol was performed by an experienced physiotherapist. SEMG signals in CES muscles were recorded bilaterally and simultaneously using a electrogoniometer during the test, before and after the intervention.

\section{Instrumentation}

\section{SEMG}

Electromyography (EMG) signals were recorded using a SEMG device (Datalog, UK). Bipolar disposal EMG surface electrodes ( $\mathrm{Ag}-\mathrm{AgCl})$ were applied on the skin, in line with muscle fibers. Inter-electrode distance was set to be $2 \mathrm{~cm}$ and electrode leads were taped on the skin [27]. SEMG data were recorded in right and left CES muscles, $2-\mathrm{cm}$ lateral to the spinous process of $\mathrm{C}-4[6,28]$. In order to decrease the impedance, the subjects' skin was shaved, abraded, and washed by water before placing the electrodes. A ground electrode was applied to the left wrist [27]. The sampling rate of EMG device was $1,000 \mathrm{~Hz}$ and the band-pass filter frequency was between $20-480 \mathrm{~Hz}$. Neck flexion and extension angles were recorded by an electrogoniometer sensor (sampling rate of $1,000 \mathrm{~Hz}$, Biometrics) synchronized with EMG data.

\section{Self-Reported Measures}

NDI questionnaire is used to show the functional changes following the treatment of CNP patients [29]. All the patients completed the Persian version of this questionnaire to assess self-reported disability, both before and after the eight weeks of intervention. It consists of 10 items, with seven items associated with activities of daily living (ADL), two items associated with pain, and one item associated with concentration. All the items of the questionnaire are scored from 0-50, with a higher score representing a higher level of disability $[29,30]$. The Persian version of NDI is a reliable and valid instrument to assess disability and functioning in CNP patients (Cronbach's alpha: 0.88 , Intraclass correlation: 0.90) [31].

VAS (which is scored from 0-10 cm, with a higher score indicating a more pain) was used to measure the intensity of neck pain. Patients were asked to determine a point along this line based on the severity of their pain during the last month. According to Bijur et al., VAS is a valid and reliable instrument to measure the severity of pain [32].

\section{Intervention}

Low-load craniocervical flexion exercises (CCFE) were performed by the subjects in crook lying position to carry out deep flexor muscles training (longus capitis and longus colli, the main cervical stabilizer muscles) presented by Jull et al. [33]. The patient's head was positioned horizontally, without any pillow under the head. The uninflated pressure sensor cuff was placed under the patient's neck and was inflated to reach a pressure of $20 \mathrm{~mm} \mathrm{Hg}$. Both the patients and the physiotherapist received visual feedback. In the first step, each patient was taught to activate the deep muscles rather than superficial muscles and perform the exercise slowly and precisely, not strongly. In the second phase, the patients were asked to perform CCFE (as, if saying yes) and try to achieve a sequence of five targets, with five increments of 2 $\mathrm{mmHg}$, to finally reach $30 \mathrm{mmHg}$. Moreover, at the beginning of the exercise, the patients' tolerance level 
was determined; it is the level at which a subject could maintain the contraction for five seconds. The treatment sessions began with a preset level and increased progressively in a range of motion (ROM), in amount of pressure and in time to maintain contraction from 5 to 10 seconds. Each level consisted of three repetitions, each lasting 10 seconds, in a predetermined level of pressure [33,34]. Throughout the treatment sessions, through touching the patient's neck, it was tried to ensure that the patient was not using rapid movements as well as movements by the superficial neck flexor muscles, such as sternocleidomastoids and scalenes. The exercise was performed for eight weeks at home, 30 minutes daily, and three sessions per week (24 sessions) under the supervision of a physiotherapist. In addition, the patients were advised to stop the practice and report it to the physiotherapist when the exercise exacerbated their pain.

\section{Data and Statistical Analysis}

Raw EMG data were collected, rectified, and filtered. Root mean square (RMS) values (time window of 50 $\mathrm{ms}$ ) were obtained to determine the parameters of FRP. FRR values were calculated through dividing the maximum EMG in the re-extension phase by the average EMG in the relaxation phase [35]. Inverse FRR $\leq$ $40 \%$ was used to determine the occurrence of FRP [26]. These measurements were carried out both before and after the intervention.

\section{Statistical analysis}

Furthermore, the distributions of VAS, NDI, FRR, and inverse FRR before and after the intervention were calculated using paired t-test. Student's t-test was used to investigate the association between the average change of VAS, NDI, and FRR and gender and duration of pain. The comparison of inverse FRR frequency after the intervention was performed using McNemar's test. The standard error of measurement (SEM) and smallest detectable change for individual patients (SDC ind $\left._{\text {d }}\right)$ was calculated using ( ) and , respectively. Data were analyzed in the Stata software (version 14) and $p<0.05$ was considered statistically significant in all the statistical tests.

\section{Results}

The mean age of the 25 patients was $31 \pm 5.2$ years (ranging from 23 to 40 years). Of all, $52 \%(n=13)$ of the patients were female. The mean weight and BMI of the NSCNP patients were $69.64 \pm 4.79 \mathrm{~kg}$ and $23.68 \pm 1.75 \mathrm{~kg} / \mathrm{m}^{2}$, respectively. Eighty percent of the patients had normal weight $(\mathrm{BMI}=18.5-25)$. In addition, the median duration of pain was 12 months (interquartile range $=6$ ).

VAS and NDI scores significantly decreased after the intervention. In addition, FRR of bilateral erector spinae increased after the intervention (Table 1). Similarly, the frequency of inverse FRR increased after the intervention (Fig. 1). The average change of VAS, NDI and FRR were not associated with gender and duration of pain in NSCNP patients (Fig. 2,3). 
Table 1

Distribution of VAS, NDI, FRR and inverse FRR among NSCNP patients

\begin{tabular}{|c|c|c|c|c|}
\hline \multirow[t]{2}{*}{ Variable } & Before Intervention & After Intervention & The average change for 2 months & \multirow[t]{2}{*}{ P-value ${ }^{*}$} \\
\hline & Mean (SD) & Mean (SD) & Mean (SD) & \\
\hline $\begin{array}{l}\text { VAS } \\
(\mathrm{mm})\end{array}$ & $26(4.08)$ & $9.4(4.4)$ & $-16.6(4.5)$ & $<0.001$ \\
\hline NDI (\%) & $31.28(7.87)$ & $14.16(5.56)$ & $-17.12(6.66)$ & $<0.001$ \\
\hline FRR & $2.37(0.68)$ & $3.02(0.71)$ & $0.65(0.39)$ & $<0.001$ \\
\hline IFRR (\%) & $45.53(12.71)$ & $34.93(8.44)$ & $-10.6(7.93)$ & $<0.001$ \\
\hline
\end{tabular}

In addition, SEM and SDC ind for VAS were lower than NDI (on a scale of 0 to 50). Thus, these indices in 25 patients, respectively, were 3.18 and 8.82 (SDC95\%= 7.78-25.42) points for VAS and 4.7 and 13.05 $(\mathrm{SDC} 95 \%=4.06-30.17)$ points in NDI.

\section{Discussion}

The present study investigated the effects of CCFE on flexion relaxation phenomenon, pain, and disability in patients with NSCNP.

Cervical stabilization exercise is a form of exercise therapy that has been suggested to restore coordination between superficial and deep neck muscles and improve impaired neuromuscular control in patients with CNP [36]. In our study, before the intervention, FRP was absent in most of the NSCNP patients and it was observed only in $36 \%$ of the patients. This finding is consistent with the results of previous studies $[6,10]$. However, after the intervention, this phenomenon was observed in $60 \%$ of the patients. Although small number of cervical studies have investigated the effect of a specific exercise protocol on FRP, some lumbar studies have assessed it. For example, Neblett et al, (2010) evaluated the impact of a protocol of seven weeks of supervised and progressive rehabilitation exercise on FRP. Moreover, their treatment plan included both stress management counseling sessions and SEMG biofeedback to trunk muscles relaxation during flexion. After the experiment, the number of patients with chronic low back pain (CLBP) who exhibited the normal FRP increased from 30-95\%. In addition, they showed that an abnormal FRP could change into a normal one via exercise [18]. Our results confirmed their findings. The results of our study revealed two points: first, it showed that FRP could be improved using a specific exercise. Second, it confirmed the main underlying mechanism of FRP (lack of core cervical stability) that was explained before. Therefore, stability exercise could optimize FRP via activating cervical deep muscles and providing adequate stability in the cervical spine $[11,26]$. Further studies are required to examine the effect of other interventions on altered FRP in NSCNP patients. 
In the present study, FRR significantly increased (from 2.37 to 3.02) after the specified stability exercise. A small number of studies have investigated the effect of a therapeutic intervention on FRR in CNP patients. According to Murphy et al., FRR decreases from 2.02 to 1.73 after implementing a 4-week exercise program [22]. It is inconsistent with our findings, which might be attributed to differences in the type of exercises. We used the specific stability exercise to activate deep stabilizer muscles, rather than superficial large muscles, because it was aimed at reducing the activity of large muscles and stimulating stabilizer muscles to normalize neuromuscular function. However, their protocol consisted of a general exercises. In addition, they showed that FRR increased after cervical manipulation and exercises [22]. Although no study has yet examined the effect of stability exercise on FRP in CNP patients, some LBP studies have shown the positive effect of these exercises on FRP.

Marshal et al. showed that Swiss ball exercise could activate deep and superficial weak abdominal muscles and increase FRR [20]. Furthermore, another study reported that lumbar stability exercise improved FRR via activating deep muscles and preventing unstable lumbar spine movement through coactivating pelvic floor muscles, deep abdominal muscles, multifidus muscles, and diaphragm [37].

The current study showed a significant improvement in VAS and NDI scores after the intervention. Our results confirmed the findings of studies by Gupta et al. [38] and Saleh et al. [36] who reported that cervical stability exercises significantly decreased the intensity of pain in CNP patients. Nonetheless, Izquirdo et al. didn't find any significant difference in VAS score between cervical stability exercise and conventional physical therapy in CNP patients [39].

Altered motor control of spine and lack of cervical stability can lead to insufficient control of the intervertebral joint movements, increased neutral zone, repeated microtrauma, and finally pain. Moreover, pain can lead to the alteration of descending drives from supraspinal centers and result in more inhibitions of impaired muscles. Therefore, this process creates a defective cycle that increases pain and disability over time [26].

The significant pain reduction after the stability exercise might be attributed to the significant improvement in FRP parameters. Stability exercise can break the mentioned defective cycle and decrease pain intensity and disability scores through activating the inhibited deep muscles, decreasing fatigability and activity of superficial muscles, and finally improving FRP [40]. In addition, Javanshir et al.'s study showed that a reduction in strength and endurance of cervical deep muscles can cause neck pain. Moreover, they reported that CCFE could lead to an increase in the strength and endurance of these muscles and reduce disability [41]. Moreover, deep neck muscles play an important role in providing proprioception in cervical spine. Therefore, CCFE can lead to proper cervical alignment, improvement in sitting posture, and a decrease in pain and disability [36]. In addition, SEM and SDC were used to assess the impact of intervention i.e. changes in FRR. Given the lower SEM and SDC for VAS than NDI, it can be said that the use of VAS to show the impact of the intervention is preferred. In addition, the present study determined FRP parameters, VAS, and NDI in both genders before and after the intervention. There were no significant differences between the genders in terms of these variables. 


\section{Limitation}

Suboccipital muscles play an important role in providing cervical proprioception and it also can be trained together with CCFE, hence, part of the effects of stability exercise may be associated with improvements in proprioception. Therefore, future studies are recommended to focus on the effect of proprioception exercise on FRP in NSCNP patients.

\section{Conclusions}

Flexion relaxation phenomenon can be reversible through performing specific stability exercise. Improvement in FRP parameters seems to be at least one of the causes of reduction of pain and disability. The stability exercise might activate the inhibited deep muscles and decreases the load on the superficial muscles. Furthermore, this study might confirm the mentioned main mechanism of FRP, and it might show the lack of stability is known as a responsible item contributing to this phenomenon.

\section{Abbreviations}

ADL

Activities of daily living

CCFE

Craniocervical flexion exercises

CES

Cervical erector spinae

CLB

Chronic low back

CNP

Chronic neck pain

DCF

Deep cervical flexors

EMG

Electromyography

FRP

Flexion relaxation phenomenon

FRR

Flexion relaxation ratio

LBP

Low back pain

NDI

Neck disability index

NSCNP

Non-specific chronic neck pain 
ROM

Range of motion

RMS

Root mean square

RTR

Relaxation time ratio

SEM

Standard error of measurement

VAS

Visual analog scale

\section{Declarations}

\section{Ethic's approvaland consent to participate}

All procedures performed in the study were in accordance with the ethical standards of the School of rehabilitation- Shahid Beheshti University of Medical Sciences research committee (approval ID= IR.SBMU.RETECH.REC.1398.405) and with the 1964 Helsinki declaration and its later amendments or comparable ethical standards. All individuals gave written informed consent before participation in the study.

\section{Consent to publish}

Not applicable. The manuscript does not contain patient identifiable data.

\section{Availability of data and materials}

The datasets used and/or analysed during the current study are available from the corresponding author on reasonable request.

\section{Competing interests}

The authors declare that they have no competing interests.

\section{Funding}

No funding was received.

\section{Authors' Contributions}

SH: Contribution to study concept and design, acquisition, analysis and interpretation of data, drafting of manuscript

$\mathrm{K}-\mathrm{K}$ : Contribution to study concept and design, acquisition, drafting of manuscript 
A-B: Contribution to analysis and interpretation of data

I: Contribution to analysis and interpretation of data, drafting of manuscript

0 : Contribution to study concept and design, acquisition, analysis and interpretation of data, drafting of manuscript

All authors have read and approved the manuscript

\section{Acknowledgments}

This paper was extracted from a physiotherapy graduate thesis. We would like to express our thanks to all the staff of School of Rehabilitation, Iran as well as to all individuals helping us in completing this research project.

\section{Authors' Information}

${ }^{1}$ Department of Physiotherapy, School of Rehabilitation, Shahid Beheshti University of Medical Sciences, Tehran, Iran. ${ }^{2}$ School of Rehabilitation, Shahid Beheshti University of Medical Sciences, Tehran, Iran.

${ }^{3}$ Physiotherapy Research Centre, School of Rehabilitation, Shahid Beheshti University of Medical Sciences, Tehran, Iran. ${ }^{4}$ Student Research Committee, Department of Epidemiology, School of Public Health and Safety, Shahid Beheshti University of Medical Sciences, Tehran, Iran. ${ }^{5}$ Physiotherapy Research Centre, School of Rehabilitation, Shahid Beheshti University of Medical Sciences, Damavand Road, Opposite to Bou Ali Hospital, Tehran, Iran.

\section{References}

1. Ghaderi F, Javanshir K, Jafarabadi MA, Moghadam AN, Arab AM. Chronic neck pain and muscle activation characteristics of the shoulder complex. J Bodyw Mov Ther. 2019;23(4):913-17.

2. Martin-Gomez C, Sestelo-Diaz R, Carrillo-Sanjuan V, Navarro-Santana M, Bardon-Romero J, PlazaManzano G. Motor control using cranio-cervical flexion exercises versus other treatments for nonspecific chronic neck pain: A systematic review and meta-analysis. Musculoskelet Sci Pract. 2019;42:52.

3. Cerezo-Téllez E, Torres-Lacomba M, Fuentes-Gallardo I, Perez-Muñoz M, Mayoral-del-Moral O, LluchGirbés $E$, et al. Effectiveness of dry needling for chronic nonspecific neck pain: a randomized, singleblinded, clinical trial. Pain. 2016;157(9):1905-17.

4. Falla $D$, Jull G, Hodges P. Feedforward activity of the cervical flexor muscles during voluntary arm movements is delayed in chronic neck pain. Exp Brain Res. 2004;157(1):43-8.

5. Falla D. Unravelling the complexity of muscle impairment in chronic neck pain. Man Therap. 2004;9(3):125-33. 
6. Maroufi N, Ahmadi A, Khatir SRM. A comparative investigation of flexion relaxation phenomenon in healthy and chronic neck pain subjects. Eur Spine J. 2013;22(1):162-8.

7. Floyd W, Silver P. The function of the erectores spinae muscles in certain movements and postures in man. J Physiol. 1955;129(1):184-203.

8. Callaghan JP, Dunk NM. Examination of the flexion relaxation phenomenon in erector spinae muscles during short duration slumped sitting. Clin Biomech. 2002;17(5):353-60.

9. Murphy BA, Marshall PW, Taylor $\mathrm{HH}$. The cervical flexion-relaxation ratio: reproducibility and comparison between chronic neck pain patients and controls. Spine. 2010;35(24):2103-8.

10. Pialasse J-P, Dubois J-D, Choquette M-HP, Lafond D, Descarreaux M. Kinematic and electromyographic parameters of the cervical flexion-relaxation phenomenon: the effect of trunk positioning. Annals of physical rehabilitation medicine. 2009;52(1):49-58.

11. Arguisuelas M, Lisón J, Doménech-Fernández J, Martínez-Hurtado I, Coloma PS, Sánchez-Zuriaga D. Effects of myofascial release in erector spinae myoelectric activity and lumbar spine kinematics in non-specific chronic low back pain: Randomized controlled trial. Clin Biomech. 2019;63:27-33.

12. Geisser ME, Ranavaya M, Haig AJ, Roth RS, Zucker R, Ambroz C, et al. A meta-analytic review of surface electromyography among persons with low back pain and normal, healthy controls. The journal of pain. 2005;6(11):711-26.

13. Falla D, O'Leary S, Fagan A, Jull G. Recruitment of the deep cervical flexor muscles during a postural correction exercise performed in sitting. 2005.

14. Falla D, O'Leary S, Fagan A, Jull G. Recruitment of the deep cervical flexor muscles during a posturalcorrection exercise performed in sitting. Man Therap. 2007;12(2):139-43.

15. Raju AS, Apparao P, Swamy G, Chaturvadi P, Mounika RG. A Comparative Study on Deep Cervical Flexors Training and Neck Stabilization Exercises in Subjects with Chronic Neck Pain. Indian Journal of Physiotherapy \& Occupational Therapy. 2019;13(2).

16. Dusunceli Y, Ozturk C, Atamaz F, Hepguler S, Durmaz B. Efficacy of neck stabilization exercises for neck pain: a randomized controlled study. J Rehabil Med. 2009;41(8):626-31.

17. Lee H-j, Park H-s, Park J-m. The Effects of Cervical Stabilization Exercise on Neck Pain, Range of Motion, and Deep Cervical Muscle Strength in Patients With Chronic Neck Pain. The Journal of Korean Academy of Orthopedic Manual Physical Therapy. 2019;25(1):9-19.

18. Neblett R, Mayer TG, Brede E, Gatchel RJ. Correcting abnormal flexion-relaxation in chronic lumbar pain: responsiveness to a new biofeedback training protocol. Clin J Pain. 2010;26(5):403.

19. Kim JH, Kim YE, Bae SH, Kim KY. The effect of the neurac sling exercise on postural balance adjustment and muscular response patterns in chronic low back pain patients. Journal of physical therapy science. 2013;25(8):1015-9.

20. Marshall PW, Murphy BA. Evaluation of functional and neuromuscular changes after exercise rehabilitation for low back pain using a Swiss ball: a pilot study. J Manipulative Physiol Ther. 2006;29(7):550-60. 
21. Shahvarpour A, Henry SM, Preuss R, Mecheri H, Larivière C. The effect of an 8-week stabilization exercise program on the lumbopelvic rhythm and flexion-relaxation phenomenon. Clin Biomech. 2017;48:1-8.

22. Murphy B, Taylor HH, Marshall P. The effect of spinal manipulation on the efficacy of a rehabilitation protocol for patients with chronic neck pain: a pilot study. J Manipulative Physiol Ther. 2010;33(3):168-77.

23. Park D-J, Park S-Y. Long-term effects of diagonal active stretching versus static stretching for cervical neuromuscular dysfunction, disability and pain: An 8 weeks follow-up study. Journal of back and musculoskeletal rehabilitation. 2019(Preprint):1-8.

24. Burnett A, O'Sullivan P, Ankarberg L, Gooding M, Nelis R, Offermann F, et al. Lower lumbar spine axial rotation is reduced in end-range sagittal postures when compared to a neutral spine posture. Man Therap. 2008;13(4):300-6.

25. Burnett A, O'Sullivan P, Caneiro JP, Krug R, Bochmann F, Helgestad GW. An examination of the flexionrelaxation phenomenon in the cervical spine in lumbo-pelvic sitting. J Electromyogr Kinesiol. 2009;19(4):e229-e36.

26. Mousavi-Khatir R, Talebian S, Toosizadeh N, Olyaei GR, Maroufi N. The effect of static neck flexion on mechanical and neuromuscular behaviors of the cervical spine. Journal of biomechanics. 2018;72:152-8.

27. Stegeman D, Hermens H. Standards for surface electromyography: The European project Surface EMG for non-invasive assessment of muscles (SENIAM). 2007.

28. Frericks $B$. The recommendations for sensors and sensor placement procedures for surface electromyography. SENIAM deliverable 8, European Recommendations for Surface Electromyography. 1999:15-53.

29. Hains F, Waalen J, Mior S. Psychometric properties of the neck disability index. J Manipulative Physiol Ther. 1998;21(2):75-80.

30. Fairbank J, Couper J, Davies J, O'brien J. The Oswestry low back pain disability questionnaire. Physiotherapy. 1980;66(8):271-3.

31. Mousavi SJ, Parnianpour M, Montazeri A, Mehdian H, Karimi A, Abedi M, et al. Translation and validation study of the Iranian versions of the Neck Disability Index and the Neck Pain and Disability Scale. Spine. 2007;32(26):E825-E31.

32. Bijur PE, Silver W, Gallagher EJ. Reliability of the visual analog scale for measurement of acute pain. Academic emergency medicine. 2001;8(12):1153-7.

33. Jull GA, O'leary SP, Falla DL. Clinical assessment of the deep cervical flexor muscles: the craniocervical flexion test. J Manipulative Physiol Ther. 2008;31(7):525-33.

34. Jull G, Falla D, Treleaven J, Hodges P, Vicenzino B. Retraining cervical joint position sense: the effect of two exercise regimes. J Orthop Res. 2007;25(3):404-12.

35. Nimbarte AD, Zreiqat M, Ning X. Impact of shoulder position and fatigue on the flexion-relaxation response in cervical spine. Clin Biomech. 2014;29(3):277-82. 
36. Saleh MSM, Rehab NI, Sharaf MAF. Effect of deep cervical flexors training on neck proprioception, pain, muscle strength and dizziness in patients with cervical spondylosis: A randomized controlled trial. 2018.

37. Park S-s, Choi B-r. Effects of lumbar stabilization exercises on the flexion-relaxation phenomenon of the erector spinae. Journal of physical therapy science. 2016;28(6):1709-11.

38. Gupta BD, Aggarwal S, Gupta B, Gupta M, Gupta N. Effect of deep cervical flexor training vs. conventional isometric training on forward head posture, pain, neck disability index in dentists suffering from chronic neck pain. Journal of clinical diagnostic research: JCDR. 2013;7(10):2261.

39. Izquierdo TG, Pecos-Martin D, Girbés EL, Plaza-Manzano G, Caldentey RR, Melús RM, et al. Comparison of cranio-cervical flexion training versus cervical proprioception training in patients with chronic neck pain: A randomized controlled clinical trial. J Rehabil Med. 2016;48(1):48-55.

40. Zabihhosseinian M, Holmes MW, Ferguson B, Murphy B. Neck muscle fatigue alters the cervical flexion relaxation ratio in sub-clinical neck pain patients. Clin Biomech. 2015;30(5):397-404.

41. Javanshir K, Mohseni-Bandpei MA, Rezasoltani A, Amiri M, Rahgozar M. Ultrasonography of longus colli muscle: A reliability study on healthy subjects and patients with chronic neck pain. J Bodyw Mov Ther. 2011;15(1):50-6.

\section{Figures}

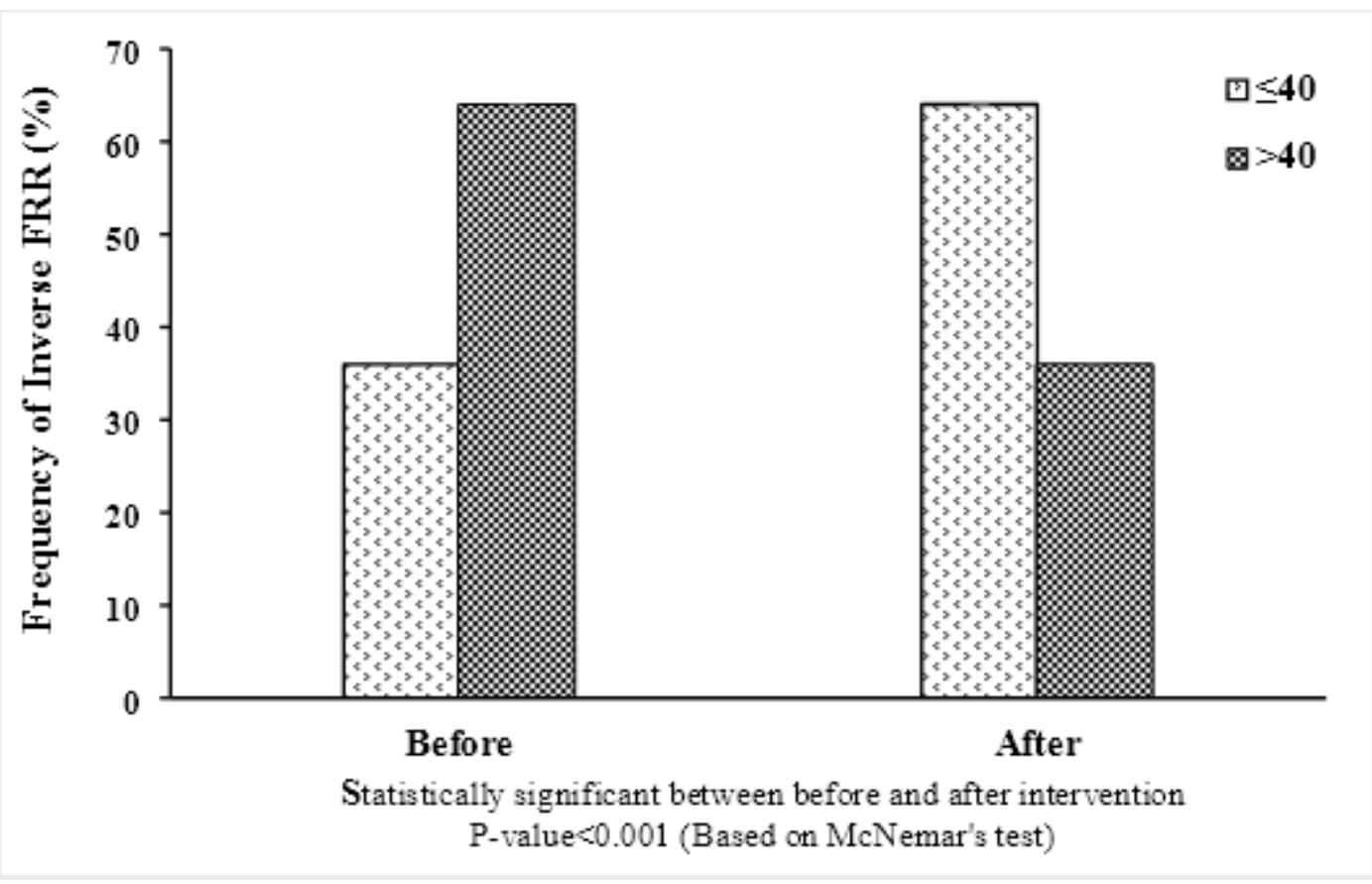

\section{Figure 1}

Frequency of inverse flexion-relaxation ratio among NSCNP patients 


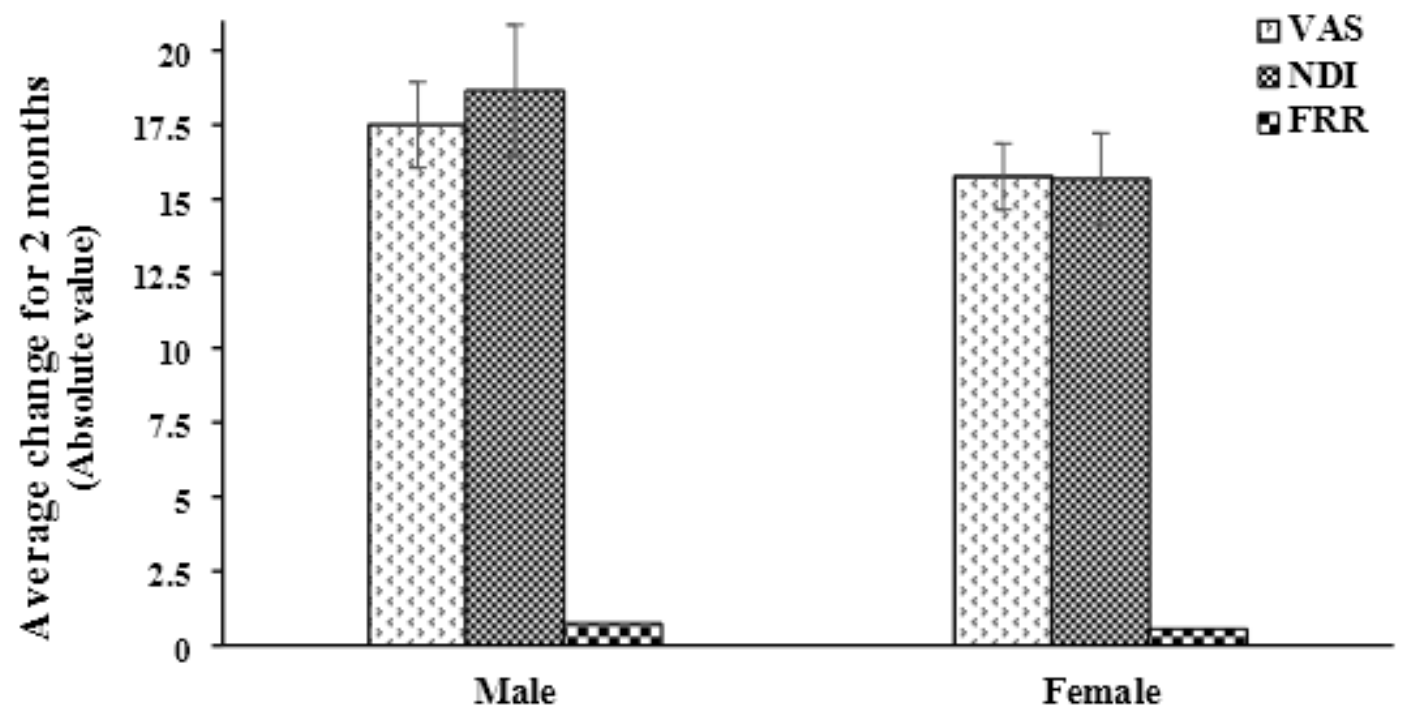

Statistically not significant between two sexes, $\mathrm{P}$-value $>0.05$ (Based on t-test)

VAS= Visual Analog Scale; NDI= Neck Diability Index; FRR= Flexion-Relaxation Ratio

Figure 2

Comparison of average change of VAS, NDI and FRR among NSCNP patients by gender

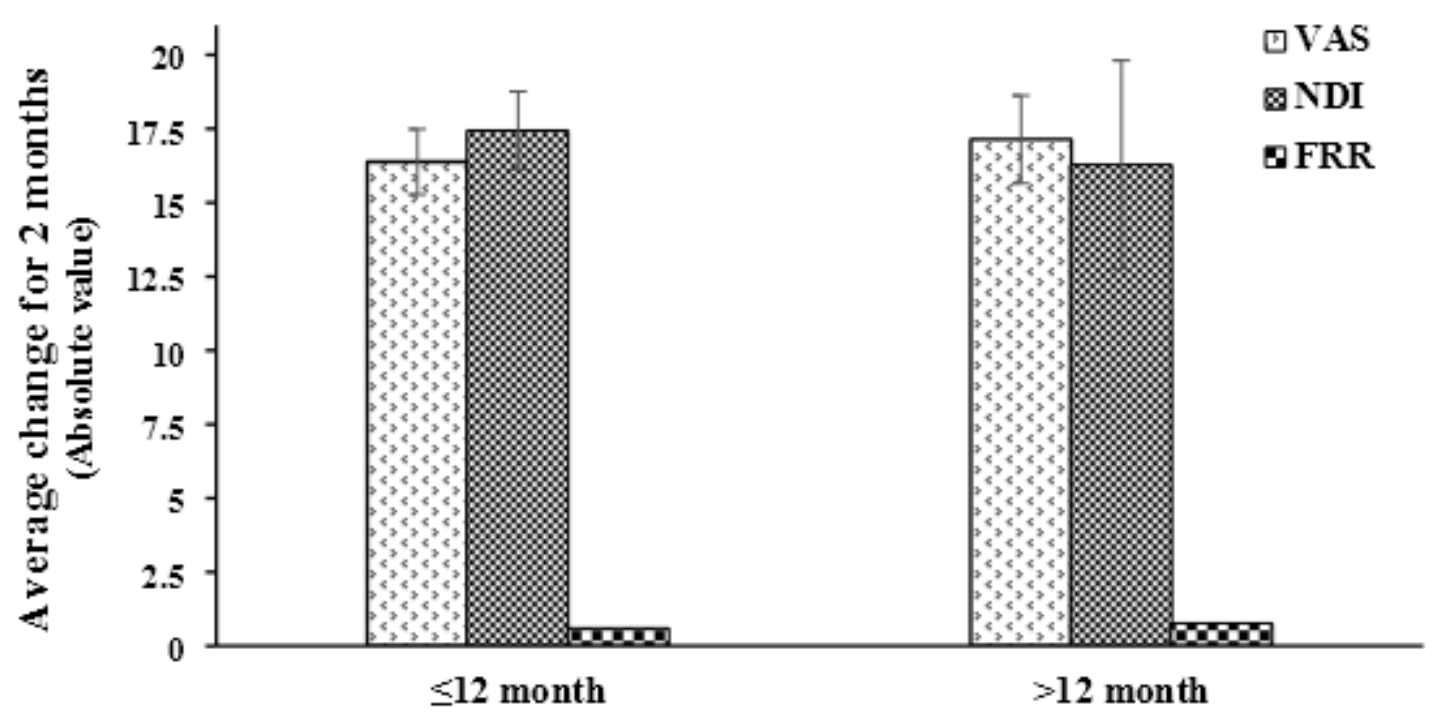

Statistically not significant between two groups, $P$-value $>0.05$ (Based on t-test) VAS $=$ Visual Analog Scale; NDI= Neck Diability Index; FRR= Flexion-Relaxation Ratio

Figure 3

Comparison of average change of VAS, NDI and FRR among NSCNP patients by duration of pain 